\title{
The Solar cycle: looking forward
}

\author{
Robert H. Cameron \\ Max-Planck-Institut für Sonnensystemforschung, 37191 Katlenburg-Lindau, Germany
}

\begin{abstract}
We discuss predictions for cycle 24 and the way forward if progress is to be made for cycle 25 and beyond.
\end{abstract}

Keywords. Sun: activity

\section{Introduction}

The level of solar activity waxes and wanes with an approximate timescale of 11 years. The amplitudes of each maximum is observed to vary strongly from cycle to cycle. Numerous attempts to predict each of the recent maxima have been made. For cycle 24 a number of predictions were collected by Pesnell(2008) and the resulting distribution is shown in Figure 1. Also shown is the distribution of cycle maxima for cycles 1-23. From this figure it appears that, as of 2008, what could be said was that the amplitude of cycle 24 wil (probably) be drawn from the same distribution as cycles 1 to 23 .

This result should not be surprising: with only 23 cycles, any number of patterns can be found in the data, and used as a basis for extrapolating the data to cycle 24. Furthermore since the predictions cover a large range it is obvious that some of them will be close to the actual level of maximum activity for cycle 24 - so the apparent success of some of these models is gauranteed (although which models will be successful was obviously not agreed on before the cycle began). Apparent success in this regard need not indicate the models will have predictive success in future cycles.

\section{The way forward}

To make progress it is essential to understand the non-linearity which is driving the solar cycle. This understanding needs to be based on observations: dynamo theory in the abstract is non-specific enough to allow predictions covering the whole range of predictions shown in figure 1 - compare for example the predictions of Dikpati \& Gilman(2006) with those of Jiang et al.(2007).

A non-linearity in this context involves the magnetic field driving a flow. For a long time it was thought that the changes were likely to be on small spatial scales where the creation of the poloidal flux from toroidal flux would be inhibited (this was called 'alpha quenching'). This form of saturation does not however work for the BabcockLeighton type of dynamo which is the favoured explanation for the global solar dynamo, see Kitchatinov \& Olemskoy(2011). Consequently the theoretical discussion has recently focussed on the dynamo saturation being due to changes in the large-scale velocity field. While a significant focus of the theoretical work has been on changes in the global meridional circulation, e.g. Karak \& Choudhuri(2012), the observations actually support only near-surfce changes to the flow field, see Cameron \& Schüssler(2010). Specifically the observations, e.g. by Gizon(2004), support a shallow, time-dependent, inflow into the active region latitudes. Cameron \& Schüssler(2012) have modelled the effect of this inflow 


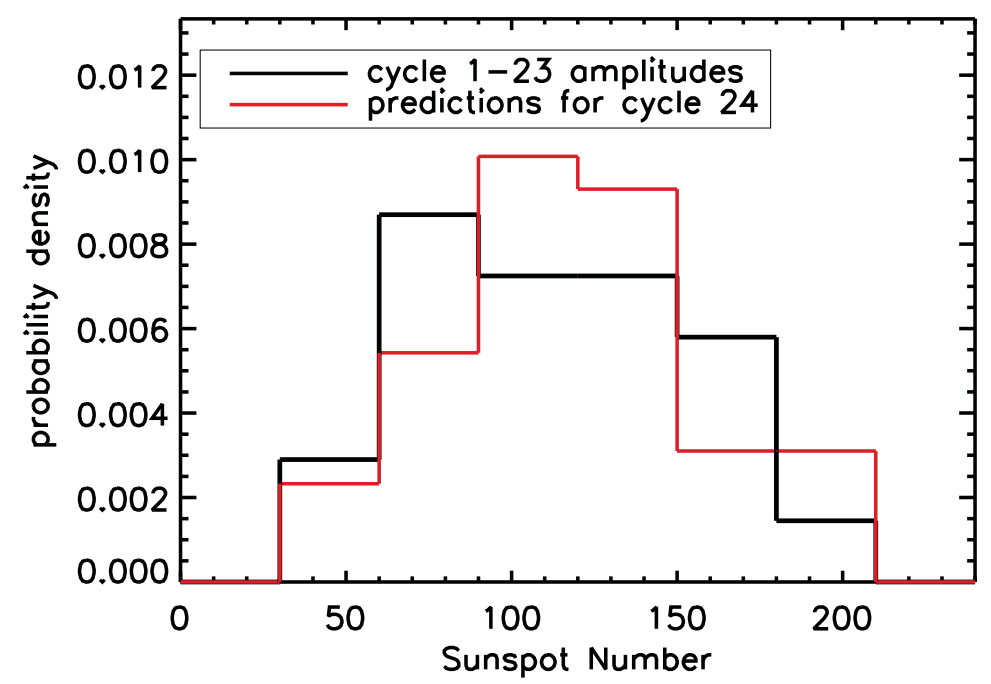

Figure 1. Distribution of predictions for the amplitude of cycle 24 given in Pesnell (2008) and of the observed amplitudes of cycles 1 to 23 .

using the surface flux transport model and find that it is able to account for most of the observed cycle-to-cycle changes in activity levels.

The inflows into the active region belt are an observationally clear nonlinearity for the dynamo which is of the correct sign and magnitude to explain most of the observed cycle-to-cycle variations. The driving of the inflows has been studied theoretically by Spruit(2003) and modelled by Gizon \& Rempel(2008): they are though to be a response to the cooling of the plasma by the excess radiance associated with regions of plage.

\section{Conclusion}

An understanding of the non-linearity which saturates the solar dynamo is a necessary precondition for believable predictions which extend beyond about $1 / 2$ a cycle - for shorter times precursors might be effective because of the Waldmeier effect, see Cameron \& Schüssler(2007). It seems that on the Sun the non-linearity occurs at the surface where it can be studied in great detail.

\section{References}

Cameron, R. \& Schüssler, M. 2007, ApJ 659, 801

Cameron, R. H. \& Schüssler, M. 2010, ApJ 720, 1030

Cameron, R. H. \& Schüssler, M. 2012, A\&BA, accepted

Dikpati, M. \& Gilman, P. A. 2006, ApJ 649, 498

Gizon, L. 2004, Solar Phys. 224, 217

Gizon, L. \& Rempel, M. 2008, Solar Phys. 251, 241

Jiang, J., Chatterjee, P., \& Choudhuri, A. R. 2007, MNRAS 381, 1527

Karak, B. B. \& Choudhuri, A. R. 2012, Solar Phys. 278, 137

Kitchatinov, L. L. \& Olemskoy, S. V. 2011, Astron. Lett. 37, 656

Pesnell, D. 2008, Solar Phys. 252, 209

Spruit, H. C. 2003, Solar Phys. 213, 1 